\title{
La misión de la universidad según la presidenta de Harvard. Breves consideraciones para Honduras
}

\section{Harvard president on the mission of the university. Some issues for Honduras}

\author{
Claudio F. Salgado* \\ claudio.student@gmail.com
}

Resumen

Drew G. Faust, Rectora de la Universidad de Harvard desde 2007, ha pronunciado diversos discursos sobre la misión de la universidad en la sociedad. De estos, hemos sacado a luz cuatro importantes ideas: que la actividad universitaria comprende tanto el presente como el pasado y futuro; que la contemplación y la formulación de hondas preguntas son intrínsecas a la universidad; que ésta contribuye al futuro bienestar social mediante el aplicado estudio y consejo; y que la enseñanza universitaria se orienta a la formación para la vida, que no solo incluye la instrucción profesional de los alumnos. En dichos discursos,
Faust también ha expresado que la universidad vive en tensión, por lo que proponemos que los administradores universitarios tienen que encontrar el justo medio de las fuerzas que tiran de ésta. Una vez expuestas dichas ideas, nos concentramos en las semejanzas de atención a las demandas sociales y en las diferencias de la formación común de pregrado, entre Faust y la legislación hondureña. Las traducciones al español son nuestras.

Palabras clave: Harvard, misión, universidad, Drew G. Faust, Honduras

\section{Abstract}

Drew G. Faust, president of Harvard University since 2007, has delivered a number of addresses on the mission of the university in society. We bring to light four of her key ideas: that the task of the

* Doctor en Gobierno y Cultura de las Organizaciones.

Recibido 8 de febrero de 2017 /Aceptado 31 de mayo de 2017.

<Paradigma> - Revista de Investigación Educativa. Año 24. No. 37 
university involves past, present and future; that contemplation and deep inquiry are intrinsic to the university; that it contributes to future well-being through careful study and advice; and that higher education is oriented towards lifelong learning, which not only includes the vocational training of students. Faust has also shown that the university lives in tension, so we suggest that university administrators should find the golden mean of it. We then focus on the similarities and differences with regard to social demands and undergraduate education, respectively, between Faust and Honduran regulations. Translations to Spanish are ours.

Keywords: Harvard, Mission, University, Drew G. Faust, Honduras

\section{Introducción}

Drew Gilpin Faust asumió la presidencia de la Universidad de Harvard en la segunda mitad de 2007. Es una reconocida historiadora de la Guerra Civil estadounidense, graduada con honores por la Universidad de Bryn Mawr. Obtuvo el doctorado en Civilización Americana en la Universidad de Pensilvania, donde también impartió clases durante veinticinco años. Posteriormente, se trasladó a Harvard, donde alcanzó la Cátedra Lincoln de Historia y el decanato del Instituto Radcliffe de Estudio Avanzado. Ha publicado seis libros, el último de los cuales, This Republic of Suffering: Death and the American Civil War, fue calificado como uno de los diez mejores de 2008 por el diario The New York Times (Universidad de Harvard, 2017).
En principio, sorprende que Faust se pronuncie acerca de la misión de la universidad, puesto que la sólida institución que dirige cuenta con una historia de más de tres siglos y destaca a nivel internacional; entre otras razones, porque Harvard posee el mayor fondo universitario del mundo, de decenas de millardos de dólares. También es sorprendente por qué Estados Unidos, donde se asienta dicha universidad, dispone de holgados márgenes de riqueza y es referente en temas de educación. Sin embargo, la crisis financiera de finales de 2000 coincidió con su ascenso a la presidencia de este reconocido centro educativo y dio pie a que reflexionara sobre la institución universitaria. En parte porque, ya en 2009, Harvard declaraba una pérdida de cerca del 
$30 \%$ de su patrimonio como efecto de esta crisis (Harvard Magazine, 2009). También porque, en su propia universidad, ha observado que solo el $14 \%$ de los alumnos cursa una carrera de humanidades, en 2016, y que ha habido una caída del $10 \%$ en las matrículas de asignaturas humanísticas de pregrado, desde 2007 (Faust y Wieseltier, 2016).

Las intervenciones de Faust sobre la misión de la universidad han tenido lugar en diversos escenarios, tanto dentro como fuera de Estados Unidos, y han perseguido el objetivo de ofrecer una mirada más completa sobre el quehacer universitario en la sociedad, que también contribuya a remontar las secuelas de la crisis de principios del siglo XXI. A nuestro entender, esta mirada puede ordenarse en función del tiempo, de la actividad universitaria, de la cooperación con la economía y del empleo. 
La vocación de historiadora ha sido de gran ayuda a Faust en el ejercicio del gobierno de Harvard, ya que, en diversas ocasiones, ha leído que todavía suele apoyarse en hechos pasados para entender el presente y para sugerir nuevas rutas al centro de educación superior que preside (Faust, 2007; Faust, 2009c; Faust, 2016).

Al respecto del tiempo y la misión universitaria, Faust ha llamado la atención sobre lo que podemos denominar presentismo, por el que se pide a los centros de nivel superior que se enfoquen solo en los problemas más urgentes de la sociedad. Si bien este pedido indica que se acude a la institución universitaria porque responde interrogantes de muy diversa naturaleza, debe tenerse en cuenta que el cometido de la universidad no se agota en el presente ni se realiza en exclusiva cuando soluciona problemas inmediatos. Ese llamado de atención se encuentra en el discurso de toma de posesión de Faust (2007), en el que se lee lo siguiente:

"La esencia de la universidad consiste en que es especialmente responsable del pasado y del futuro, no única ni primariamente del presente. La universidad no trata de los resultados del próximo trimestre, ni mucho menos de lo que el estudiante ha logrado al momento de graduarse. Trata de enseñanzas que forman el resto de la vida, de enseñanzas que transmiten el patrimonio de siglos, de enseñanzas que modelan el futuro. La universidad ve tanto hacia atrás como hacia adelante, de modo que puede -e incluso debe de-entrar en conflicto con las solicitudes e inquietudes presentes. Las universidades se comprometen con lo eterno, y este compromiso tiene efectos que no podemos predecir y, a menudo, tampoco podemos medir" (Faust, 2007).

Según este discurso, la universidad no debería centrarse exclusivamente en el pasado, como si fuera un museo en donde se exhiben antiguas colecciones; ni proyectarse únicamente al futuro, para eludir dificultades contemporáneas, aunque tampoco tendría que obsesionarse con las agitaciones del presente. En realidad, se trata de que los problemas cotidianos no hagan quitar la mirada de las riquezas del pasado ni de las esperanzas de lo que está por venir. Esto quiere decir que, para lograr una perspectiva completa de la universidad, habría que reevaluar la importancia que otorgamos a los distintos tiempos de la vida humana, tendríamos que buscar la integración armónica de pasado, presente y futuro y su aplicación a las tareas universitarias. Dicho de otra manera, a Universidad Pedagógica Nacional Francisco Morazán, de Honduras, se comparten bajo términos de la Licencia Creative Commons: Se permite que otros puedan descargar las obras y compartirlas con otras personas, siempre y cuando se reconozca su autoría, pero no se pueden cambiar de ninguna manera ni se pueden utilizer comercialmente. 
los centros de educación superior cumplirán a cabalidad su papel una vez que, en el presente, se beneficien del pasado recibido, sean "servidores de la viva tradición" (Faust, 2007) y trabajen con vistas al futuro.

La universidad debería discrepar de las solas demandas actuales, porque es posible que se vuelva "miope" (Faust, 2009b; Faust, 2012c; Faust, 2012d) y, consecuentemente, incapaz de dar consejo al que lo solicita. Por el bien de la misma sociedad, la universidad debe marcar prudente distancia de los acontecimientos recientes, pues, de lo contrario, se dejarían de lado aspectos como la profundidad y la extensión del saber, según Faust (2010) pronunciaba en el Trinity College de Dublín:

... nos arriesgamos a perder de vista las preguntas más generales, los tipos de investigación que motivan el enfoque crítico, que moldean la perspectiva humana, que promueven el incesante escepticismo y curiosidad sin límites desde los que, a menudo, surgen nuestros más hondos pensamientos. El enfoque muy estrecho en el presente puede venir a expensas del pasado y del futuro, del largo plazo que siempre ha sido preocupación especial de la educación superior (Faust, 2010).

Esta concepción de la universidad, que armoniza pasado, presente y futuro, al menos requiere el ejercicio de la paciencia, puesto que profesores y alumnos necesitan tiempo suficiente para asimilar las enseñanzas recibidas y también necesitan ser pacientes mientras van profundizando en las cuestiones que se les plantean. La sociedad en general también debe ser paciente con la universidad y aceptar que ésta le sirve mejor cuando se respetan sus modos. No se trata de resignación, sino que es una paciencia activa, que ayuda a que los miembros de la universidad perseveren hasta el final y a que la sociedad colabore con ellos en la solución de las preguntas que les formulan.

\section{En relación con su actividad: la universidad se detiene a contemplar}

Otro riesgo que Faust ha identificado acerca de la universidad, vista solo como medio para zanjar dificultades actuales, yace en que se prescinda de su necesaria actividad contemplativa:

Un modelo demasiado instrumental de la universidad pierde el genio de su capacidad. Acorta la zona de paciencia y contemplación que la universidad crea en un mundo casi abrumado por los estímulos. Rebaja 
su papel de investigador de preguntas fundamentales en un mundo que se apresura a solucionar los problemas más urgentes. Necesitamos ambos (Faust, 2010).

La práctica universitaria no puede calificarse de dirigida, puesto que, para bien o para mal, no cuenta con un listado de pasos a seguir a rajatabla. La universidad se destina, eso sí, al más largo plazo, a las inquietudes más ocultas y misteriosas del hombre y el universo y a “... los descubrimientos que tardan -y duran-décadas e incluso siglos. $\mathrm{Ni}$ las ineludibles preguntas de la investigación humanística ni el sinuoso camino de la exploración científica, que finalmente produce innovaciones y descubrimientos, pueden encajarse perfectamente en predecibles cronogramas ni presupuestos"' (Faust, 2009b).

Si tuviéramos que puntualizar la contemplación de la universidad, habría que decir que ésta consiste en detenerse a pensar sobre todo en temas de gran dificultad y que es una tarea que requiere mucho esfuerzo y genera cansancio, a pesar de que no puede asegurarse que tendrá resultados inmediatos ni susceptibles de comercializarse. Pero, curiosamente, los universitarios por vocación suelen decir que este natural cansancio es superado con creces por el disfrute de aprender y de transmitir lo que saben.

La actividad contemplativa se vincula con la enseñanza cuando ayuda a que los estudiantes se admiren de su entorno, amplíen sus perspectivas y enfoquen interrogantes de manera disciplinada. En dicho vínculo, se genera una verdadera tradición académica, en tanto que los mayores obsequian lo mejor de sí y van preparando a las nuevas generaciones, y los jóvenes van poniendo mucho de su parte para crecer en saber. La contemplación incluye el hacer y las labores manuales porque, mientras se realiza, los universitarios organizan jornadas, dictan clases, elaboran proyectos de investigación, publican informes, etc.

En este caso, la contemplación ha de apoyarse en la virtud de la humildad, como nos explicaba Faust (2007) cuando discurría sobre el lema que anima a su centro educativo -veritas, verdad-:

"La verdad es una aspiración, no una posesión. Nuestra universidad y todas las que se inclinan por el debate y la libre investigación incitamos e importunamos a los que admiten certezas incuestionables. Debemos la Universidad Pedagógica Nacional Francisco Morazán, de Honduras, se comparten bajo términos de la Licencia Creative Commons: Se permite que otros puedan descargar las obras y compartirlas con otras personas, siempre y
cuando se reconozca su autoría, pero no se pueden cambiar de ninguna manera ni se pueden utilizer comercialmente. 
comprometernos con la incómoda posición de duda, con la humildad de creer que siempre se puede saber más, enseñar más, entender más" (Faust, 2007).

En la universidad, son frecuentes las ocasiones en que se necesita ejercitar dicha humildad, porque suele ocurrir que los materiales didácticos son insuficientes; que no hay colegas ni alumnos para desarrollar investigaciones y que, a pesar del deseo por querer saber, los propios conocimientos no alcanzan los objetivos propuestos. Pero hay que decir que la humildad también tiene un sentido positivo, pues apunta a que pueden verse con nuevas luces textos leídos años atrás y que vendrán otros -el indispensable relevo generacional- para proseguir los trabajos. También puede ampliarse la idea de humildad que nos ofrece Faust, ya que dicha virtud incluso tiene un aspecto cualitativo que la presidenta de Harvard no mencionó, pues es razonable aducir que siempre puede saberse mejor, enseñarse mejor y entenderse mejor. Los profesores pueden comprobarlo al examinar las grandes diferencias entre la forma en que impartían clases al inicio y en los últimos años de su carrera docente.

Con humildad, habría que aceptar la posibilidad de que las instituciones de educación superior se hayan aislado de la sociedad o se hayan vuelto sordas a sus peticiones, lo que denotaría no solo la indiferencia de parte de los centros de nivel superior a las demandas sociales, sino que también representaría que, de hecho, estos han abandonado la importante tarea universitaria de contemplar. Aunque lo más justo sería determinar si profesores y alumnos no han contestado por negligencia o porque están abstraídos en la contemplación, que, como hemos dicho, requiere de tiempo suficiente y mucho esfuerzo.

En este punto, conviene añadir que la sociedad está igualmente llamada a practicar la humildad, en especial cuando tiene que considerar que la misión contemplativa de la universidad implica retroceder, como sugiere la presidenta Faust (2012b):

[La educación] es dar un paso atrás del urgente presente para ver más clara y críticamente y ser capaz de imaginar un mundo diferente; un mundo que construimos no solo para hoy y mañana, sino para el mañana de décadas y generaciones futuras. [La cursiva es nuestra] (Faust, 2012b).

<Paradigma> - Revista de Investigación Educativa. Año 24. No. 37 
Esto quiere decir que la sociedad ha de aceptar que los universitarios por vocación no solo se detienen a pensar, sino que, en algunas ocasiones, incluso dan marcha atrás y desandan el camino recorrido. Humildemente, la sociedad también debe reconocer que las instituciones de educación superior no siguen su ritmo y que pueden tardar en disipar las dudas que les plantea. Pero tiene que saber diferenciar entre las universidades sordas e indiferentes y las que están absortas en problemas complejos.

\section{En relación con el desarrollo económico: la universidad prevé el futuro}

Entre las diversas presiones que la economía ejerce sobre la educación superior, las más importantes radican en que se pide a la universidad que prepare jóvenes para los sectores e industrias que aumenten la riqueza nacional y que encauce su quehacer a la mejora de la competitividad, sobre todo porque, hoy en día, se perciben con más claridad las amenazas de la globalización y la libre circulación de capitales. Es de suponer que Faust conoce la dinámica global y sus efectos sobre las distintas poblaciones, pero la presidenta de Harvard ha tratado de mostrar que la universidad sirve mejor a la actividad económica cuando lo hace según su propia especialidad, que consiste en poner las bases del futuro progreso social. Ante distinguidos invitados de la Asia Society, de India, Faust leía lo siguiente:

Solemos estar ansiosos por la investigación que promueve el desarrollo económico. Sin embargo, me preocupa que esta ansiedad nos motive a asumir el corto plazo, el bajo riesgo y que desluzcamos las más hondas interrogantes y la perspectiva humana, que deben guiar el desarrollo sustentable y de largo plazo. En la carrera por hacer frente a crisis acuciantes y presentes en salud y abastecimiento de agua, y en encontrar soluciones a necesidades energéticas y a agricultura sostenible -vitales para el bienestar futuro- me preocupa que, por encargarnos de éstas, corramos el riesgo de subestimar el libre escepticismo y la curiosidad que, a menudo, generan nuestros más hondas reflexiones... (Faust, 2012a).

Faust no deja de lado necesidades como alimentos, energía, sanidad o agua, sino que las coloca en su lugar al reconocer que son esenciales para la humanidad. Pero, incluso en un país emergente -como es Indiala presidenta de Harvard hace hincapié en que el mayor aporte de la universidad a la economía se concreta en estudios de carácter prospectivo. la Universidad Pedagógica Nacional Francisco Morazán, de Honduras, se comparten bajo términos de la Licencia Creative Commons: Se permite que otros puedan descargar las obras y compartirlas con otras personas, siempre y
cuando se reconozca su autoría, pero no se pueden cambiar de ninguna manera ni se pueden utilizer comercialmente. 
Esto quiere decir que la universidad debe examinar el actual concepto de desarrollo y buscar las maneras de alcanzarlo, bajo criterios de factibilidad y conveniencia. Ha de ponderar si el modelo de desarrollo económico es adecuado según factores políticos, jurídicos, morales y religiosos; debe investigar cuáles son los medios disponibles para atajar el desarrollo previsto y cuáles tienen que potenciarse. La universidad incluso ha de emitir juicios sobre aspectos más delicados, como pueden ser la finalidad del desarrollo mismo, si hay que generalizarlo y en qué medida es bueno para el país.

En ese sentido, las instituciones de educación superior también deben llevar la batuta en el proceso que podríamos llamar el "desarrollo del desarrollo", que consiste en la identificación de las áreas que los modelos de innovación social no abarcan en la actualidad. Se trata de que dichas instituciones piensen con rigor y mucha serenidad acerca de lo que hoy en día se da por sentado en materia de progreso de los seres humanos, cuyas reflexiones podrían discrepar de las teorías y fundamentos que se enseñan en las aulas universitarias. Por ejemplo, y tomando como base el discurso de Faust en India, cabe la pregunta de si el estudio de las más hondas interrogantes acerca del hombre forma parte de la concepción de progreso de los principales índices sociales de este siglo. La universidad tendría que valorar si dichas interrogantes están presentes o ausentes de lo que se entiende por desarrollo económico, y, llegado el momento, argumentar en favor de su inclusión en los debates académicos.

El análisis prospectivo de la economía debe ir unido a la crítica social, porque puede ocurrir que la universidad se entere de que las consultas que se le hacen no aciertan en los verdaderos problemas que aquejan a los ciudadanos. Esta crítica implica el llamado a que se replanteen los fines del país y los medios con que estos se persiguen, ya que quizá no sean conformes con el bien de la totalidad de la población; y es posible que dicha crítica acarree molestias, sobre todo entre los que dirigen a la ciudadanía. En ese caso, hay que dejar claro que la universidad se expresa en orden a mejores rutas y destinos.

Según lo expuesto en este apartado, debe insistirse en que los centros de educación superior no necesariamente siguen el ritmo de la sociedad a la que sirven, ya que no solo dan pasos atrás, sino que -como hemos visto aquí- también han de acelerar la marcha para luego poner sobre aviso al conjunto de la sociedad. 


\section{En relación con el empleo: la universidad educa para la vida}

Estrechamente unida a la economía, se encuentra la solicitud a las universidades de que enseñen principalmente técnicas y herramientas que faciliten la salida laboral de los alumnos. Dicha solicitud es comprensible en un entorno que busca recuperarse de la grave crisis financiera de la década pasada, que ha reducido las tasas de incorporación al mercado de trabajo y ha aumentado la deserción escolar porque no puede abonarse el importe de las matrículas. Sin embargo, la propuesta de Faust sobre la enseñanza en el ámbito universitario es mucho más generosa que el mero empleo de los recién graduados, pues, a su criterio: "La educación superior puede ofrecer a los individuos y sociedades profundidad y amplitud, que tienden a faltar en el irremediablemente miope presente. Los seres humanos necesitan significado, entendimiento y perspectiva tanto como empleos" (Faust, 2009b).

Basada en su larga experiencia académica y la centenaria tradición de Harvard, Faust defiende que las denominadas "artes liberales" son la manera más propia de la universidad para satisfacer las necesidades de amplitud, profundidad, significado y empleos antes dichas. Y lo hace con las siguientes razones:

En primer lugar, las artes liberales destacan en amplitud porque se componen de las humanidades, las ciencias sociales y las ciencias experimentales. En Harvard, dichas artes liberales conforman el Programa de Educación General, que representa del 25\% al 33\% de los planes de estudio de todas las carreras de pregrado de esa universidad (Faust, 2010).

En segundo lugar, las artes liberales sobresalen por su profundidad porque es habitual que se planteen interrogantes de gran calado: las humanidades ayudan a los alumnos de Harvard a reflexionar sobre la condición humana, ya que en ellas se ponderan cuestiones como "¿Qué es bueno? ¿Qué es justo? ¿Cómo sabemos que algo es verdadero?" (Faust, 2014) y se indagan roles humanos, como los de "padres, enamorados, [y] amigos" (Faust y Wieseltier, 2016). En las ciencias experimentales también se da la oportunidad de que los alumnos formulen grandes interrogantes, concretamente acerca de los orígenes del universo y del hombre (Faust, 2014). Otras preguntas de las artes liberales se hallan en una intervención de Faust en el Boston College: “¿Cómo deberíamos vivir? ¿Quién quiero ser hoy y mañana?... ¿Adónde espera ir [la humanidad] y dónde debería de ir?" (Faust, 2012d). la Universidad Pedagógica Nacional Francisco Morazán, de Honduras, se comparten bajo términos de la Licencia Creative Commons: Se permite que otros puedan descargar las obras y compartirlas con otras personas, siempre y cuando se reconozca su autoría, pero no se pueden cambiar de ninguna manera ni se pueden utilizer comercialmente. 
En tercer lugar, las artes liberales aventajan en el cultivo del significado, según la presidenta de Harvard: "Este tipo de entendimiento [, el significado,] yace en la esencia de la universidad. El significado trata de interpretación. Es comprender el mundo y nosotros mismos no solo mediante la innovación y el descubrimiento, sino que también por medio del reinventar, del reexaminar, del reconsiderar disciplinado. [...] El significado consiste en recordar lo que habíamos olvidado, en un nuevo contexto; es oír y ver lo que está justo delante de nosotros, que antes no percibíamos; es la sabiduría que debe de revolverse y despertarse cada vez, incluso entre los eruditos" (Faust, 2010).

Resulta, pues, que el significado requiere de práctica para agudizar la percepción de realidades a nuestro alcance. Es un ejercicio que puede calificarse de paradójico porque aspira a encontrar novedades en cosas que ya estaban presentes. Es como si dichas novedades yacen en el que ve y oye más que en el objeto o que el propio objeto se revela a medida que el espectador lo mira.

El texto de Faust también deja entrever que el significado se constituye de dos dimensiones: una objetiva, que trata sobre la comprensión abstracta del entorno y de la humanidad en general; y una dimensión subjetiva o personal, en la que se examinan las implicaciones de lo que nos rodea para cada uno de nosotros. Esta dimensión subjetiva tiene lugar cuando la lectura da paso a la introspección, pues el lector se ve plasmado en algún personaje de la novela, comedia o relato histórico.

En este punto, conviene señalar que la importancia de las artes liberales en la Universidad de Harvard no se debe a una mera repetición de sus tradiciones, sino que radica en un compromiso vivo y actualizado con los que ellos consideran la mejor educación -incluso tras la crisis financiera-, como Faust pronunciaba durante los actos de graduación de la promoción de 2009: "[Los profesores y directivos de Harvard] Hemos puesto énfasis en que la mejor educación es la que cultiva hábitos intelectuales, espíritu analítico, capacidad de juicio y de indagación, que les permitirá adaptarse a cualquier circunstancia o tomar cualquier dirección profesional" (Faust, 2009a).

En Bombay, Faust volvía a referirse a dicha mejor educación, aunque de modo más extenso: "La mejor educación justamente consiste en la libertad -en el imperativo- de pensar, de imaginar, de objetar y de 
cambiar. Las universidades orientadas a la investigación existen para innovar con una honda corriente de humanidad. Nuestro propósito no solo es crear mejores empleos sino mejores vidas: inspirar el pensamiento creativo, que distinguimos de la información o del conocimiento recibido; poner en entredicho supuestos, abrir nuestras mentes y extender nuestro común sentido de posibilidad" (Faust, 2012a).

Esto da a entender que, a nivel institucional, Harvard está convencida de que las artes liberales representan la mejor educación para el siglo XXI, porque éstas ejercitan el razonamiento y generan buenos hábitos, que pueden seguir perfeccionándose en cualquier ámbito familiar o profesional. Las artes liberales también sirven para educar para la vida, ya que con esa capacidad de razonamiento podemos reconsiderar la propia existencia y los ambientes en que se desarrolla, e igualmente para acostumbrar a los jóvenes a discurrir situaciones desde muchos puntos de vista, a veces opuestos; a pronunciarse en favor o en contra de determinados temas y a hacerlo con rigurosos argumentos.

Como decíamos más arriba, Faust defiende que las artes liberales satisfacen diversas necesidades individuales y sociales, entre ellas la urgente inquietud por el futuro laboral de los estudiantes, tal y como llegó a escribir en el New York Times:

El objetivo [de las artes liberales] no consiste en preparar a los alumnos para un empleo en concreto sino para muchos puestos de trabajo a lo largo de la vida, y que necesitan de amplitud y profundidad de comprensión, que solamente se consiguen por medio de las artes liberales y su exposición del vasto saber humano (Faust, 2015).

De este texto de la presidenta Faust podemos sacar a luz dos valiosas reflexiones. La primera consiste en averiguar de qué maneras los hábitos intelectuales cultivados por medio de las artes liberales contribuyen al trabajo profesional. Sin ánimo de agotarlas, al menos indicamos las siguientes respuestas: el ejercicio de dichos hábitos podría ayudar a imaginar nuevas soluciones a problemas empresariales; a discernir causas de efectos operativos; a valorar proyectos desde niveles comerciales, financieros o técnicos; a comunicarse de forma clara y con un estilo cuidado; a percatarse del entorno en que se llevan a cabo los negocios y a ponerse en el lugar de otro -jefe o compañero- con miras a entenderlo. Es decir, que se pondrían en práctica actividades de pensar, imaginar, objetar, cambiar y de indagar roles humanos, que mencionaba Faust. la Universidad Pedagógica Nacional Francisco Morazann, de Honduras, se comparten bajo términos de la Licencia Creative Commons: Se permite que otros puedan descargar las obras y compartirlas con otras personas, siempre y cuando se reconozca su autoría, pero no se pueden cambiar de ninguna manera ni se pueden utilizer comercialmente. 
La segunda reflexión versa sobre el riesgo de que la universidad quiera estar tan cerca de los empleadores, y tan preocupada por la inserción laboral de sus estudiantes, que instruya a estos últimos en las técnicas de sectores económicos en boga, sin tener en cuenta que dichos sectores pueden desaparecer en unos años. La excesiva orientación al mercado de las carreras universitarias, a corto plazo, puede facilitar que los alumnos consigan trabajo, pero, a largo plazo, podría estar reduciendo sus posibilidades de cambiar de industria y empleo.

\section{La "tensión creativa" de la universidad y su evaluación}

Como hemos podido ver, la misión de la universidad en la sociedad se desarrolla en una "tensión creativa" (Faust, 2012d), habida cuenta de que la universidad no puede estar tan cerca de las incógnitas del presente que la vuelva miope, ni tan lejos que no pueda percibirlas; y de que se dispone a contemplar misterios y oscuridades de la vida humana, aunque esta práctica sea ardua, ya que bien puede decirse que la verdad encuentra a los universitarios sumidos en su búsqueda. La universidad también vive bajo tensión porque ha de preparar el futuro del país y de sus estudiantes mientras que atiende a la economía y el empleo a corto plazo; y porque depende de la colaboración de la sociedad, aunque algunas veces deba señalarle los errores que comete. Otras tensiones que Faust (2012d) ha indicado, y que pueden ser objeto de futuras publicaciones, se refieren al conocimiento y el carácter, a la verdad intelectual y la verdad moral, y a la exploración desinteresada y el fiel compromiso social.

Los administradores universitarios deben ser responsables de dos importantes tareas. La primera consiste en que, cuando uno de los extremos tira con más fuerza a la universidad, ellos tienen que corregir el rumbo de la institución que dirigen, de manera que ésta continúe sus encargos en un prudente medio. Dichos administradores también pueden adoptar resoluciones que integren las dimensiones a las que nos hemos aproximado en este trabajo, ya que la armónica integración de esas dimensiones resultaría eficaz contra situaciones de estrés que la tensión provoca en los miembros de la universidad. La segunda tarea de estos administradores radica en que deben de mostrar los anchos horizontes de la institución universitaria, para evitar malentendidos con la sociedad, en especial en este momento en que la crisis financiera ha obligado a recortar presupuestos y ha provocado numerosos desempleos en la población. En otras palabras, en diversos foros, las autoridades de la educación superior tendrían que exponer una apropiada y completa 
idea de la universidad, de las tensiones que en ésta tienen lugar y de los bienes a los que aspira.

En ese sentido, Faust se ha pronunciado sobre la evaluación de universidades en función del primer empleo de los graduados. En una columna de opinión del New York Times ponía como ejemplo su misma carrera profesional:

Me gradué del Bryn Mawr College en 1968 y mi primer empleo fue en el Departamento de Vivienda y Desarrollo Urbano. Mi primer salario era bajo, pero me inspiré en el movimiento de derechos civiles y en la guerra contra la pobreza para tener el servicio público por vocación importante. Después estudié el posgrado, me incorporé al claustro de profesores de la Universidad de Pensilvania y, con el tiempo, me convertí en presidenta de la Universidad de Harvard. ¿Acaso debería juzgarse a Bryn Mawr con base en mi primer salario en ese departamento? (Faust, 2013).

Faust ha objetado dicha evaluación porque el salario inicial es un indicador muy limitado de la misión de la universidad, ya que no tiene en cuenta ni la huella que se desea marcar en los estudiantes, por medio de las artes liberales, ni su responsabilidad con el pasado y el futuro de la humanidad, ni las inesperadas vueltas de la vida; y parece conceder primacía al rápido éxito económico en vez de otros aspectos más esenciales, como el servicio a los demás.

La presidenta de Harvard sí se ha mostrado partidaria de la evaluación universitaria, cuando se corresponde con su naturaleza compleja y sus propósitos (Faust, 2007). Por una parte, las evaluaciones de carácter externo tendrían que incluir todas las perspectivas y tensiones de los grandes cometidos de las instituciones de educación superior. Dichas evaluaciones deberían ser capaces de responder de qué maneras puede medirse un organismo que resguarda las riquezas del pasado y avista el mañana, cuál es la combinación adecuada entre el modelo instrumental y el modelo contemplativo de la universidad, cuál son las mejores disposiciones para que los universitarios busquen la verdad, cómo valorar la sabiduría de profesores experimentados y el potencial de profesores jóvenes; cuándo la universidad debe de retroceder y cuándo debe de adelantarse, cuál es la medida justa entre la promoción del desarrollo contemporáneo y la investigación del "desarrollo del desarrollo", por qué es mejor la instrucción técnica que la educación en la Universidad Pedagógica Nacional Francisco Morazán, de Honduras, se comparten bajo términos de la Licencia Creative Commons: Se permite que otros puedan descargar las obras y compartirlas con otras personas, siempre y
cuando se reconozca su autoría, pero no se pueden cambiar de ninguna manera ni se pueden utilizer comercialmente. 
artes liberales, o viceversa; qué es éxito, qué es un graduado exitoso, etc. Por otra parte, la tendencia de la universidad a indagar y a reexaminar sus actividades sugiere que ésta tiene sus propias formas de evaluarse, por lo que es muy probable que, a lo interno, surjan voces de profesores y alumnos inconformes con el camino de la universidad. En lugar de acallar a estos inconformes, los administradores tienen una tercera importante tarea, que comprende el favorecer las condiciones para que la universidad despliegue su natural función crítica, incluso consigo misma.

\section{Breves consideraciones para Honduras}

Una vez expuesto el vasto panorama de la misión de la universidad en la sociedad según Faust, queremos enfocar este trabajo en lo que las leyes nacionales prescriben acerca de las relaciones entre la sociedad y la universidad en Honduras. Con este fin, específicamente nos basaremos en la Constitución de la República (CRH), la Ley de Educación Superior (LES), las Normas Académicas de Educación Superior (NAES) y el Reglamento General de la Ley Fundamental de Educación (RLFE).

Para comenzar, resulta conveniente señalar que las normativas vigentes sí ordenan que la universidad preste atención a las demandas sociales, las cuales pueden ir desde el apoyo a la transformación de la sociedad hondureña (CRH, art. 160; LES, art. 3; LES art. 6; NAES, art. 7), a la solución de los problemas de la comunidad, la región y el país (NAES, art. 6; NAES, art. 31) y hasta la cooperación al desarrollo sostenible nacional (RLFE, art. 65).

En las Normas Académicas de Educación Superior se ofrecen lineamientos más concretos sobre cómo las universidades hondureñas dan respuesta a las peticiones sociales, pues éstas indican que los centros de educación superior responden con la enseñanza que imparten, formal e informalmente; con propuestas de mejora del país que son fruto del estudio de la realidad nacional y con la divulgación de los avances más destacados en materia de ciencia, tecnología y cultura (NAES, art. 9). Estas maneras de responder coinciden con las funciones de docencia, investigación y extensión, que tradicionalmente se asocian con la educación superior.

Si nos concentramos en la función de enseñanza, encontraremos que las leyes nacionales dictan que "formar profesionales al más alto

<Paradigma> - Revista de Investigación Educativa. Año 24. No. 37 
nivel" y que "contribuir a la formación integral del ciudadano hondureño con vistas al desarrollo integral del país", son dos de los principales fines de la educación superior (NAES, art. 6). El reciente Plan Estratégico del Sistema de Educación Superior de Honduras 2014-2023 también recoge esos dos fines, cuando menciona que el conjunto de la educación superior participa del desarrollo del país mediante la "formación integral de profesionales y ciudadanos responsables y éticos" (Dirección de Educación Superior, 2014, p. 26).

Las normativas al uso disponen que la formación integral se consiga mediante la armónica unión de conocimientos teóricos, del contexto hondureño, éticos y de deberes profesionales (LES, art. 3). La responsabilidad por esta formación integral recae sobremanera en la universidad, que representa la categoría institucional más elevada en el sistema de educación superior y que tiene como obligaciones cardinales el preparar los profesionales que el país requiere y el transmitir a los estudiantes universitarios una así denominada "cultura superior" (NAES, art. 50).

Tanto los fines de la educación superior como los medios con los que se alcanza la formación integral desembocan en el plan de estudios, en el que se plasma de manera sintética la enseñanza universitaria según todas sus vertientes, profesional, humanística, científica y tecnológica, y demás aspectos pedagógicos (NAES, art. 82). Concretamente en las carreras de pregrado, la legislación en vigor declara que los planes de estudio deben componerse de una Formación General y de una Formación Específica. La Formación General tiende a ofrecer un panorama amplio y unitario sobre el hombre, el mundo y la sociedad y a estimular la perspicacia y el sentido crítico de los alumnos (NAES, art. 86). En esta formación, las NAES puntualizan que obligatoriamente se incluyan las asignaturas de Español, Filosofía, Sociología e Historia de Honduras y prescriben que dicha Formación General no exceda de ocho asignaturas del total del currículo (NAES, art. 87). Por su parte, la Formación Específica de pregrado tiene una clara orientación al mercado laboral, ya que su objetivo es el desarrollo de conocimientos, habilidades $\mathrm{y}$ actitudes para profesiones concretas (NAES, art. 88).

En este punto, surge el interrogante de si el plan de estudios de pregrado es capaz de moldear la conducta cívica de los alumnos, que es un fin de la educación superior hondureña, y si las asignaturas de Formación General son suficientes para estimular el razonamiento crítico la Universidad Pedagógica Nacional Francisco Morazán, de Honduras, se comparten bajo términos de la Licencia Creative Commons: Se permite que otros puedan descargar las obras y compartirlas con otras personas, siempre y cuando se reconozca su autoría, pero no se pueden cambiar de ninguna manera ni se pueden utilizer comercialmente. 
que se espera de un graduado universitario, puesto que dicha Formación General suele confundirse con cursos nivelatorios o introductorios, en los que difícilmente se ahonda en las áreas del saber propuestas, de Español, Filosofía, Sociología e Historia de Honduras.

Si hacemos una comparación entre la manera en que se organiza el plan de estudios en Honduras y la propuesta de Faust sobre enseñanza universitaria, encontramos una gran diferencia en cuanto al peso de las asignaturas comunes a las carreras de pregrado, pues en Harvard éstas constituyen hasta un tercio del currículo mientras que en Honduras éstas no deben de pasar de ocho, con lo cual resulta imposible que las universidades locales se planteen una educación en artes liberales o un Programa de Educación General, como se conoce en Harvard.

Sin embargo, consideramos que debe explorarse dicho Programa de Educación General por dos razones. La primera es que un prestigioso centro educativo contempla que las artes liberales siguen siendo el medio idóneo de la universidad para dar cauce a las demandas sociales y para sentar las bases técnicas, humanas y cívicas de los alumnos. La según razón yace en que quizá no se ha tenido en cuenta que, al enfatizarse la Formación Específica, se corre el riesgo de perder el lenguaje común de los universitarios. Esa pérdida podría obstaculizar el diálogo inter, multi y transdisciplinar al que se aspira en los centros de educación superior del siglo XXI, oscurecería la unidad que da nombre a la propia institución universitaria y, finalmente, también repercutiría en los trabajos de los mismos profesionales universitarios.

\section{Conclusiones}

Desde hace una década, Drew Gilpin Faust, presidenta de la Universidad de Harvard, se ha expresado acerca de la misión de la universidad y, más concretamente, sobre las maneras en que las universidades pueden ayudar a la solución de diversos problemas sociales, especialmente en materia de economía y empleos. Para los primeros problemas, Faust ha propuesto que la universidad se encargue de la reflexión serena acerca del desarrollo de base amplia, que incluya tantas iniciativas para paliar necesidades básicas como aspectos más esenciales de la condición humana. Para los segundos problemas, Faust ha insistido en que las artes liberales -conocidas en Harvard como Programa de Educación General- son la mejor vía para instruir

<Paradigma> - Revista de Investigación Educativa. Año 24. No. 37 
profesionalmente a los estudiantes universitarios y para prepararlos para el resto de la vida.

En Honduras, las normativas vigentes también ponen énfasis en que las universidades deben de responder a las demandas sociales, sobre todo en lo concerniente al desarrollo y transformación de la sociedad. Esas mismas normativas establecen que las respuestas de las universidades se traducen básicamente en la educación de los jóvenes, en el estudio del contexto nacional, en la elaboración de propuestas de reforma de la sociedad y en la divulgación de los adelantos de todas las áreas del saber.

En lo que atañe a la educación superior, la legislación hondureña prescribe que la universidad contribuya a la formación integral de los estudiantes, la cual comprende aspectos intelectuales, éticos, cívicos, deontológicos y técnico-profesionales. A nivel de pregrado, la misma legislación ordena que el plan de estudios compagine la Formación Específica y la Formación General. De esta última, se estipula que tiene por fin el "formar profesionales con sentido crítico y conscientes de sus responsabilidades públicas y humanas, para una mejor contribución a la transformación de la realidad nacional". Sin embargo, también se manda que esa Formación General no constituya más de ocho asignaturas del plan de estudios de pregrado.

En ese sentido, la educación basada en artes liberales o Programa de Educación General -que propone Faust- no puede implantarse en Honduras, porque dicho programa representa del 25\% al 33\% del currículo de todas las carreras de pregrado de Harvard, que es mucho más que las ocho asignaturas de Formación General que se permiten en las universidades nacionales.

Cabe preguntarse si, con la Formación General, realmente se alcanza a incentivar el pensamiento crítico; a transmitir la cultura superior, que según la legislación hondureña es lo más propio de la universidad; y a concienciar a los estudiantes de pregrado acerca de sus responsabilidades específicamente ciudadanas, habida cuenta de que la Formación Específica los orienta solo a los conocimientos, habilidades y actitudes necesarios para el trabajo. la Universidad Pedagógica Nacional Francisco Morazán, de Honduras, se comparten bajo términos de la Licencia Creative Commons: Se permite que otros puedan descargar las obras y compartirlas con otras personas, siempre y cuando se reconozca su autoría, pero no se pueden cambiar de ninguna manera ni se pueden utilizer comercialmente. 


\section{Referencias Bibliográficas}

Constitución de la República de Honduras. (1982).

Dirección de Educación Superior de la UNAH. (1992). Normas académicas de la educación superior. Recuperado de https://des.unah.edu.hn/ dmsdocument $/ 576$

Dirección de Educación Superior de la UNAH. (1994). Ley de educación superior, Reglamento general de la ley, Normas académicas del nivel de educación superior. Recuperado de-https://des.unah.edu.hn/dmsdocument/577

Dirección de Educación Superior de la UNAH. (2014). Plan Estratégico del Sistema de Educación Superior de Honduras. Recuperado de-https:// des.unah.edu.hn/dmsdocument/2611

Faust, D. (2007). Installation Address, Unleashing our Most Ambitious Imaginings. Recuperado de http://www.harvard.edu/president/speech/ 2007/installation-address-unleashing-our-most-ambitious-imaginings

Faust, D. (2009a). 2009 Baccalaureate Service, Improvisation and the Art of the Possible. Recuperado de http://www.harvard.edu/president/speech/ 2009/2009-baccalaureate-service-improvisation-and-art-possible

Faust, D. (2009b). The University's Crisis of Purpose. The New York Times. Recuperado el 5 de diciembre de 2016, de http://nyti.ms/1IqwWbt

Faust, D. (2009c). Remarks by Drew Gilpin Faust at the General Education Launch Event. Recuperado de http://www.harvard.edu/president/speech/ 2009/remarks-by-drew-gilpin-faust-general-education-launch-event

Faust, D. (2010). The Role of the University in a Changing World. Recuperado de http://www.harvard.edu/president/speech/2010/roleuniversity-changing-world

Faust, D. (2012a). Universities in a Changing World. Recuperado de http://www.harvard.edu/president/speech/2012/universities-changingworld

Faust, D. (2012b). Challenges of Higher Education. Recuperado de http:/ Lwww.harvard.edu/president/speech/2012/challenges-higher-education

<Paradigma> - Revista de Investigación Educativa. Año 24. No. 37

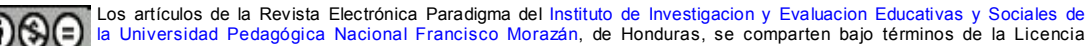
Creative Commons: Se permite que otros puedan descargar las obras y compartirlas con otras personas, siempre y cuando se reconozca su autoría, pero no se pueden cambiar de ninguna manera ni se pueden utilizer comercialmente. 
Faust, D. (2012c). 2012 Commencement Speech. Recuperado de http:// www.harvard.edu/president/speech/2012/2012-commencement-speech

Faust, D. (2012d). Scholarship and the Role of the University: Remarks at the Boston College Sesquicentennial. Recuperado de http://www.harvard.edu/ president/speech/2012/scholarship-and-role-university-remarks-bostoncollege-sesquicentennial

Faust, D. (2013). Don't Judge a College's Value by Graduates' Paycheck. The New York Times. Recuperado de http://nyti.ms/UNXf4j

Faust, D. (2014). 2014 Commencement Speech. Recuperado de http:// www.harvard.edu/president/speech/2014/2014-commencement-speech

Faust, D. (2015). 2 College Presidents, on Higher Education. The New York Times. Recuperado de http://nyti.ms/1I0GsRG

Faust, D. (2016). Morning Prayers: "Historians All". Recuperado de http://www.harvard.edu/president/speech/2016/morning-prayershistorians-all

Faust, D. y Wieseltier, L. (2016). Aspen Ideas Festival: Do We Need to Rescue the Humanities? Recuperado el 11 de enero de 2017, de http:// www.aspenideas.org/session/do-we-need-rescue-humanities

Harvard Magazine. (2009). \$11 Billion Less. Recuperado de http:// harvardmagazine.com/2009/11/harvard-endowment-update

Poder Ejecutivo de Honduras. (2014). Reglamento General de la Ley Fundamental de Educación. Recuperado de http://www.acnur.org/t3/ fileadmin/Documentos/BDL/2016/10625.pdf

Universidad de Harvard. (2017). Biography. Recuperado de http:// www.harvard.edu/president/biography la Universidad Pedagógica Nacional Francisco Morazán, de Honduras, se comparten bajo términos de la Licencia Creative Commons: Se permite que otros puedan descargar las obras y compartirlas con otras personas, siempre y
cuando se reconozca su autoría, pero no se pueden cambiar de ninguna manera ni se pueden utilizer comercialmente. 